\title{
La productividad y tipos de turismo en la provincia de Los Ríos
}

DOI: https://doi.org/10.33262/ap.v2i3.34

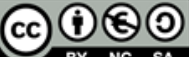

\section{Productivity and types of tourism in the province of Los Rios}

\author{
Christian Rivera García. ${ }^{1}$ \& Glenda Parra Cerezo. ${ }^{2}$
}

\begin{abstract}
.
This research aims to take advantage of agricultural productivity to expand the possibilities of tourism ventures in the province, based on the bifurcated tourism typology throughout the Fluminense territory, tourism ventures converge in starting a business investment with direct or financed capital, that involves human talent with its multiple resources. Market analysis is essential to assemble the initiative within low-interest state policies with payment facilities, seeking strategic allies is key to undertaking large-scale community tourism initiatives, favoring associativity and cultural coexistence on natural and cultural resources in micro business growth. This sector becomes vulnerable when business ideas are worked independently, except for the purchasing power possessed by the person or family group that make up the project, technical advice from public and private companies encourages the participation of well-organized peasant organizations, with Goals and objectives established previously, informal entrepreneurship is transformed into unfair work towards entrepreneurship duly recognized in the tax institutions, encountering limitations of economic growth, but demanding new initiatives and innovations in promotions of commercial products of first necessity towards the market identified as a goal.

${ }^{1}$ Universidad Técnica de Babahoyo Facultad de Ciencia Jurídicas, Sociales y de la Educación, Babahoyo, Ecuador. crivera@utb.edu.ec

${ }^{2}$ Universidad Técnica de Babahoyo Facultad de Ciencia Jurídicas, Sociales y de la Educación, Babahoyo, Ecuador.gparra@utb.edu.ec
\end{abstract}


Keywords: productivity, entrepreneurship, tourism, investors, resources.

\section{Resumen.}

Esta investigación tiene como objetivo aprovechar la productividad agrícola para ampliar las posibilidades de emprendimientos turísticos en la provincia, en base a la tipología de turismo bifurcada en todo el territorio fluminense, el emprendimientos turísticos confluyen en iniciar una inversión de negocio con capital directo o financiado, que involucre al talento humano con sus múltiples recursos. El análisis de mercado es fundamental para ensamblar la iniciativa dentro de políticas estatales a bajo interés con facilidades de pago, buscar aliados estratégicos es clave para emprender iniciativas turísticas comunitarias a gran escala, favoreciendo la asociatividad y la convivencia cultural sobre los recursos naturales y culturales en crecimiento micro empresarial. Este sector se vuelve vulnerable cuando se trabaja independientemente las ideas de negocios, a excepción del poder adquisitivo que posea la persona o grupo familiar que conforman el proyecto, el asesoramiento técnico desde la empresa pública y privada incentiva la participación de organizaciones campesinas bien organizadas, con metas y objetivos establecidos con anterioridad, el emprendimiento informal se transforma en trabajo desleal hacia emprendimiento debidamente reconocidos en las instituciones tributarias, encontrando limitaciones de crecimiento económico, pero exigiendo nuevas iniciativas e innovaciones en promociones de productos comerciales de primera necesidad hacia el mercado identificado como meta.

Palabras claves: productividad, emprendimiento, turismo, inversionistas, recursos.

\section{Introducción.}

Ampliar las posibilidades de crecimiento económico en determinadas zonas geográficas del Ecuador, es un reto donde convergen los elementos de la planificación turística, en busca de la sostenibilidad en escenarios rurales con gran potencialidad de desarrollo, enfatizada en la cultura de los pueblos milenarios acorazadas en sus costumbres, tradiciones y saberes ancestrales, las múltiples oportunidades se transforman en pequeños emprendimientos familiares y comunitarios que despegan por sus propias iniciativas.

En Ecuador existen desigualdades marcadas en sus tres regiones continentales, el desarrollo esta encajonada al aprovechamiento racional de la productividad agrícola de las zonas, los cambios políticos y las normativas estatales solo prevén estancamiento en los medios de producción y aumento desordenado en los patrones de consumo sin la planificación mancomunada de organizaciones públicas y privadas, el problema está en resolver la integración de estos sectores a las verdaderas necesidades de la población civil, en especial los sectores más pobres del país. En este aspecto se destaca el papel de las políticas sociales y fundamentalmente aquellas que alienten la economía social como vínculo de inclusión e integración social a partir de encontrar una alternativa productiva con la que se pueda enfrentar los problemas de desempleo y exclusión (Villar, 2008). 
Los nuevos modelos de gestión, en las diferentes zonas administrativas del país coinciden en el aprovechamiento racional de los recursos naturales, esa demanda turística responde a tendencias globalizadoras que diagnóstica, diseña, ejecuta y valora iniciativas productivas de gran calado, proporcionando reorganizar actividades para el disfrute y distracción de los viajeros. (Rivera, C. et al. 2019). Las dimensiones de aprovechamiento se enfocan en agua, suelo y gente irrigados por las empresas públicas, empresas privadas con el acompañamiento y asesoramiento de la academia en múltiples niveles de organización. El aspecto rural, aglutina a la comunidad reflejada en la convivencia y la asociatividad como claves del desarrollo participativo, conduciendo sus capacidades a la conservación de los recursos naturales y culturales propios de la zona. Dentro de esta perspectiva, en el contexto actual, cobran relevancia las acciones para llevar adelante políticas para las PyMes y los microemprendimientos, esto aparece tanto en la literatura especializada sobre el desarrollo local como en el discurso político- económico actual (Cimoli, M. 2005).

La presente investigación permea la problemática de las escasas plazas de trabajo, la migración en la zona litoral, que resquebraja la unión familiar y sus lazos de corresponsabilidad social, los pocos asentamientos turísticos, la explotación del suelo por los monocultivos, el incontrolable uso de agroquímicos que contaminan el agua, suelo y aire, causas que desemboca en el escaso aprovechamiento de la productividad agrícola y la limitada actividad turística en la provincia de Los Ríos.

Esta situación podría precisar de metas constitutivas y proyectistas de cambio socio-cultural a futuro, trabajo mancomunado y la reducción de la brecha de desempleo, ampliar fuentes de trabajo en la región, ampliar los micro emprendimiento como alternativa de desarrollo local, asesorar la siembra de diversas semillas con el acompañamiento de la empresa pública y la reutilización de abonos orgánicos en mejoras de la calidad de los productos, aprovechar la productividad agrícola para ampliar las posibilidades de emprendimientos turísticos de la provincia, es el objetivo principal de este trabajo.

Las unidades de observación reflejan el interés de la población en formar agrupaciones de cooperación comunitaria, administrando sus propios productos agrícolas y contribuyendo al incremento de visitantes por convivir entre reliquias culturales de intercambio étnico local, regional, nacional e internacional, atrayendo nuevos inversionistas hacia la actividad turística de esta zona.

Los impactos seria positivos, las comunidades debelarían sus capacidades de administración, mediante talleres participativos hacia nuevos emprendimientos y la transformación de la calidad de vida poblacional, en detalle se precisan los impactos, políticos, económicos y científicos.

\section{Desarrollo.}


La provincia de Los Ríos sitiada por el aporte hídrico de gran magnitud, sin tener salida al océano Pacífico, cuenta con trece cantones de gran productividad agrícola y ganadera, rodeada de más de 500 atributos fluviales entre ríos, riachuelos, cascadas, esteros, humedales, entre otros. La riqueza cultural de su territorio despierta el interés de la población local, nacional e internacional por disfrutar de sus costumbres, tradiciones y saberes ancestrales, compaginados con la diversidad de cultivos típicos de la zona donde sobresalen, el cacao, el arroz, el banano, el verde, la naranja, la sandía, y muchos otros productos que se relacionan directamente con los recursos naturales distinguiendo y clasificando las actividades turísticas en cada ciudad de la capital fluminense como por ejemplo: ecoturismo, turismo de aventura, turismo comunitario, agroturismo, turismo cultural, turismo religioso, etnoturismo, turismo de naturaleza, turismo de convenciones y eventos, turismo de negocios, esta tipología y modalidades de turismo ennoblecen a la población ratificando la popularidad de sus recursos naturales y culturales, este contexto dominante de la región ha estado en la mira de grandes corporaciones financieras nacionales y extranjeras que buscan la oportunidad de invertir en esta pujante provincia, Los Ríos con su capital Babahoyo es la Meca de las exportaciones de banano, cacao, arroz, como principales productos de consumo mundial.

Las actividades derivadas de estos cultivos obligan a la población a planificar en nuevos negocios colaborativos y complementarios en la línea aritmética y geométrica de producción, esta cultura emprendedora cambia las necesidades por oportunidades favoreciendo la construcción de cualidades, conocimientos, habilidades y valores fundamentales en un ser humano creativo, emprendedor e innovador crea en las personas iniciativas propias de ideas programáticas que se transforman a posteriori en proyectos sociales, el emprendedor diseña, lanza y pone en funcionamiento un negocio, sus capacidades tienden a dar frutos constantes de ideas en planificación, desarrollo, organización y manejo de un negocio, junto con sus respectivos riesgos, con el fin de obtener una ganancia.

Torrent-Sellens (2014), señala que el emprendimiento innovador es la forma más correcta de generar nueva actividad empresarial o transformar la existente a través de los procesos de valoración, desbordamiento o filtro del conocimiento. Además, los procesos de emprendimiento innovador encajan bien con las distintas tipologías de conocimiento y con los efectos de red, tan importantes para explicar la entrada, la supervivencia y el éxito de las nuevas iniciativas de negocio en la economía global del conocimiento.

Olivos, P. (2015), plantea que "emprender es más que gestionar una empresa, es adaptarse a los nuevos escenarios de mercado que la economía impone”. Es prácticamente imposible hablar de este asunto y no relacionarlo con el término innovación ya que son indispensables y cuando se unen la organización sólo tiene que celebrar.

Para Drucker (1985), la innovación es la herramienta específica de los emprendedores; el medio por el cual explotar el cambio como una oportunidad para un negocio diferente. Las empresas que realizan este tipo de actividades (esfuerzos innovativos) son consideradas 
innovativas mientras que se denomina innovadoras a las que logran alcanzar resultados (es decir, introducir innovaciones).

El emprendimiento constituye una de las principales actividades que desarrollan las personas en procura de conseguir los recursos que garanticen una vida digna; y a través de dignificar su existencia tributan a la generación de empleo (Escobar Terán,Mendoza Vargas, Boza Valle \& Alcivar Saltos, 2019).

Sin embargo, existe otro tipo de emprendimiento que asume el apellido de subsistencia, son los que predominan en las microempresas de la región latinoamericana. Al menos en América Latina en el año 2010 "el 75\% de los empresarios se ubican en la categoría de microempresas, generando menos de 5 puestos de trabajo, los mismos que representan el $40 \%$ de los trabajadores asalariados del sector privado". (Borja, S. 2018, p 19).

Según los datos tomados del Instituto Nacional de Estadísticas y Censos de Ecuador (2011), en la actualidad el $44 \%$ de empleo en el país andino se deben a las iniciativas del emprendimiento y las microempresas, su cifra apenas, garantizan un ingreso del $24 \%$ a la economía nacional, siendo el $21 \%$ de las pequeñas empresas, el $18 \%$ de las medianas y el $37 \%$ de la gran empresa (Borja, 2015).

Las microempresas de América Latina han adquirido tal relevancia que, en la ctualidad, según el estudio realizado por Avalos \& Murillo (2013), representan el 97\% de la población empresarial, generan el $70 \%$ de los empleos formales y, según algunas estimaciones representan entre el 30\% y $60 \%$ del PIB.

Frente a esto, el estudio realizado por Jaramillo, Morales, Escobedo \& Ramos (2013), explica que un elemento imprescindible para elevar la productividad de la microempresa radica en la capacitación e innovación de los emprendedores.

Este último aspecto, el financiero, no pocas veces ralentiza la posibilidad de desarrollo de estas organizaciones, pues muchos emprendedores temen a la hora de pedir créditos o establecer algún tipo de acuerdo con grandes empresas para insertarse en el mercado de una manera más competitiva y destacada. Para Bustamante \& Cabrera (2017), existe mucho desconocimiento en relación "a temas de finanzas del negocio con finanzas familiares no pudiendo evidenciarse de manera adecuada en la rentabilidad que pueda generar el micronegocio" (p.25).

El debate académico ha venido alimentando los cambios de enfoques de política, observándose Una evolución desde programas de promoción de la empresarialidad muy focalizados en aspectos Parciales del fenómeno (por ejemplo, la capacitación de emprendedores), hacia una concepción estratégica más integral (Hegglin, D. 2013). En consecuencia, las iniciativas más recientes suelen incluir un conjunto más articulado de 
acciones destinadas a cooperar sobre planos tales como el de la cultura, el sistema educativo y las competencias emprendedoras, la promoción de redes empresariales de apoyo y el financiamiento a nuevas y pequeñas empresas, entre otras (Cimoli; Kim 2005).

\section{Inversionistas nacionales y extranjeros en proyectos turísticos.}

\section{Empresarios internacionales interesados por invertir en Ecuador.}

Inversionistas y representantes de empresas de los Estados Unidos visitan el Ecuador con el objetivo de establecer contactos y recibir información que les permita definir futuros planes de inversión en áreas que, como el turismo, ofrecen claras oportunidades para concretar sus iniciativas, en diversas zonas de nuestro País.

- Som

- Apex Group

- Fortune International Realty

- China Telecom

- Supreme Suites

- Crea USA

- Aecom

- Boyd Jenerette

- Guil Holding

- Asset Recyclers

- Extraordinaria Partners

Son parte de las quince empresas que enviaron a sus representantes en búsqueda de oportunidades de negocios en el Ecuador, atendiendo a una invitación de la embajadora de Ecuador en los EE.UU., Nathalie Cely.

El Ministerio de Turismo espera consolidar la ejecución de más de 30 proyectos turísticos provenientes de capitales nacionales y extranjeros, con una inversión que hasta el momento registra USD 1,600 millones.

Esto como resultado del crecimiento económico que ha experimentado el país en los últimos 7 años; la sustancial mejora en infraestructura; la creciente llegada de turistas que recibe el Ecuador y la atención que reciben los inversionistas del Programa de Atracción de Inversiones en Turismo.

El programa contempla el posicionamiento de Ecuador como un destino de inversiones turísticas a nivel internacional, la mejora del clima de inversiones del sector y un permanente acompañamiento al inversionista desde que inicia el interés por invertir en el país hasta la puesta en marcha de la inversión. 
Actualmente los inversionistas reciben de un calificado equipo de profesionales del Ministerio información, la elaboración de agendas con socios estratégicos, asesoría en los beneficios que están amparados en el Código de la Producción, como la exoneración del pago al Impuesto a la Renta y la firma de contratos de inversión con el Estado que le brinda estabilidad tributaria.

\section{Proyectos.}

Entre los proyectos más relevantes que se desarrollan y se desarrollarán en el país durante los próximos años constan:

Karibao de la empresa Pronobis; Planta hotelera que se construirá en el sector El Engabao, cantón Playas-Guayas, con una inversión de USD 700 millones que se desarrollará durante 8 años. De esta inversión el 60\% es nacional y el 40\% extranjera. Isabel Noboa Pontón una de las inversionistas de este proyecto, es una empresaria ecuatoriana conocida por su incursión en los negocios agrícola, inmobiliario, industrial, comercial y turístico, a través del Consorcio Nobis uno de los grupos empresariales más grandes de Ecuador - del que Noboa es fundadora y presidenta ejecutiva. En la actualidad es considerada una de las mujeres empresarias más importantes de América Latina.Así mismo, Isabel Noboa tiene trayectoria en iniciativas de filantropía.

Swiss Towers, estará en el Malecón 2000 de Guayaquil, demandará una inversión de USD 120 millones. El 60\% es inversión nacional y el 40\% extranjera, la primera piedra se colocó en enero del 2015. Estas dos torres del Swiss Towers, es un proyecto hotelero cuyo inversionista tiene la firma del arquitecto ecuatoriano Christian Wiese. Así es concebido, el complejo Swiss Tower con el primer Swissôtel en Guayaquil será la estructura más alta del país. Con sus 40 pisos, el hotel se levantará en medio de los dos edificios más grandes de la urbe en la actualidad: el complejo de oficinas The Point, de $137 \mathrm{~m}$ de altura; y el ya icónico La Previsora, con $133 \mathrm{~m}$ de altura.

Hotel Eurobuilding Express de la empresa venezolana ZARPECA S.A. Está ubicado en las áreas aledañas del Aeropuerto Internacional Mariscal Sucre. Tiene prevista la construcción de 147 habitaciones, con una inversión de USD 20 millones. El movimiento de tierras comenzó en 1982, estimando la apertura del primer Eurobuilding Hotel en 1985; el hotel fue administrado por Promociones Eurobuilding de Venezuela, una de las compañías operadoras de hoteles en España; el propietario era Eurobuilding International, cuya capital en ese momento era mixta, es decir, extranjera, nacional y del estado.

Hotel Wyndham Gran Cóndor Quito de la compañía de negocios y propiedades GRAN CONDOR CONPROGRA S.A, Empresa filial de Pronobis. Estará en Tababela, Aeropuerto Mariscal Sucre, tendrá una inversión de USD 17 millones, su inauguración está prevista para finales de 2015. Isabel Noboa Pontón(inversor) presidenta del Consorcio 'Pronobis' indicó 
que la inversión generará plazas de trabajo: "al menos un millar de fuentes de trabajo directas e indirectas se originarán gracias a la edificación del hotel para nuestros hermanos ecuatorianos".

El diseño del hotel está inspirado en el ave insigne del Ecuador, el Cóndor, que desde el cielo podrá ser visibilizado por los pasajeros como elemento distintivo de la llegada al país. Jorge Albán, alcalde (E) del Distrito Metropolitano de Quito, manifestó que "la expectativa es enorme para el sector hotelero porque generará una buena imagen turística”.

Hotel Ibis Quito y Guayaquil, de la empresa Ecuaresorts S.A., bajo la reconocida cadena hotelera francesa Accor, invertirá USD 20 millones.

El proyecto, bajo el modelo de franquicia, lo ejecutará Accor junto a su inversor aliado en el país andino, Ecuaresort S.A., y prevé tener en operación los primeros hoteles en las ciudades de Quito y Guayaquil entre 2016 y 2017. "Este proyecto contribuye al posicionamiento del sector turístico como estratégico para el país", valoró el presidente de Ecuaresort S.A., José Ochoa, al precisar que "la operación hotelera se realizará bajo los estándares de Accor, pero bajo la administración de la empresa ecuatoriana".

Giardini (Boutique Hotel y Residences), administrado por MMG Ecuador. Constructora: FRACTALES CIA. LTDA. Es un edificio amigable con el medio ambiente, inteligente, moderno y funcional que estará en el Malecón de Guayaquil. Ha sido calificado por la exclusiva cadena europea Design Hotels para albergar al primer «hotel de diseño» del Ecuador, en su sección residencial ofrece suites y departamentos de lujo. Tenderá una inversión de USD 14 millones.

Sheraton Cuenca de la compañía ESTRATEGAS INMOBILIARIOS S.A. Proyecto hotelero ubicado junto al Centro de Convenciones Mall del Río, cuenta con un monto de inversión estimado USD 13 millones. Luigi Lantermo empresario ecuatoriano que junto a un grupo de emprendedores ecuatorianos impulsa el proyecto Giardini, afirma que cree en el país y que por lo tanto crear fuentes de trabajo y oportunidades para mucha gente, es uno de los motores que les motiva a impulsa a invertir en el país. "Creo en mi país, creo en la gente de mi país, creo en la unión público-privada, creo que tenemos un país maravilloso, que, si lo trabajamos en armonía y unión, dará trabajo y futuro a las nuevas generaciones, que son nuestros hijos", añade Lantermo tras señalar que hoy en día la competitividad es fundamental en turismo y que es necesario prepararse para competir de igual a igual.

Estas iniciativas de inversionistas nacionales y extranjeros procuran en nuestro país la reactivación económica apoyados en la actividad turística, están creando nuevos polos de desarrollo mercantil e invitan a la población a capacitarse, perfeccionarse y asesorarse en productos complementarios de encadenamiento comercial, que elaboren nuevos, bienes, 
servicios, productos y procesos, dentro de un sistema turístico de competitividad a gran escala nacional.

\section{Metodología.}

Tablas 1. La productividad y tipos de turismo en la provincia de Los Ríos

\begin{tabular}{|c|c|c|}
\hline Indicadores dominantes & $\begin{array}{c}\text { Porcentaje de aceptación } \\
\text { prioritaria }\end{array}$ & $\begin{array}{l}\text { Acciones procedimentales } \\
\text { en beneficio de la } \\
\text { creatividad e innovación }\end{array}$ \\
\hline $\begin{array}{c}\text { Nuevos emprendimientos } \\
\text { virtuales vigorizan la } \\
\text { actividad turística }\end{array}$ & $51.5 \%$ & $\begin{array}{c}\text { Asesoramiento } \\
\text { tecnológico, debería incluir } \\
\text { equipo informático }\end{array}$ \\
\hline $\begin{array}{c}\text { La competencia } \\
\text { gastronómica es desleal }\end{array}$ & $48.5 \%$ & $\begin{array}{c}\text { Financiamiento } \\
\text { microempresarial, para } \\
\text { servicios complementarios }\end{array}$ \\
\hline
\end{tabular}

Fuente: Elaboración propia.

El enfoque cuantitativo permite realizar una investigación de carácter descriptivo, el impacto de la creación de micro emprendimientos momentáneos y limitados, sugiere un trato especial en la economía familiar, es un fenómeno escasamente estudiado que dirige las necesidades poblacionales a soluciones parches, con excepciones del manejo adecuado de las tecnologías de información y comunicación en el menor tiempo posible, estos emprendimientos posibilitan la generación de economía auxiliar, para la obtención de los resultados se solicitó llenar una encuesta dirigida a micro emprendedores de una zona específica, el muestreo intencional fue realizada a 100 personas emprendedoras, particularmente en negocios de alimentación.

\section{Resultados.}

Se aplicaron encuestas a micro emprendedores entre 25-40 años de edad, que habitan en zonas rurales, que se sienten desesperados por la situación económica de país y buscan una solución alternativa a la economía actual. Los encuestados mencionan que necesitan regular sus actividades mediante asesoramiento técnico y conocer las ventajas, desventajas de cómo incursionar en un nuevo negocio productivo complementando las actividades agrícolas del quehacer diario. La utilización de los sembríos agrícolas sería una solución a corto plazo, las innovaciones se dan por curiosidad en relación a un mercado meta y un producto interesante.

\section{Discusión.}

La estructura de emprendimiento radica en la complementariedad de negocios como actividades claves, socios, relaciones con los clientes, productos de calidad y amigable con 
el medio ambiente, con seguridad en la limpieza y manipulación de alimentos, atención con calidad como propuesta de valor agregado, incluida la estructura de segmentos en el mercado en posición de anclaje de servicios complementarios y no tan competitivos entre los mismos socios, la fuente de ingresos está sujeta hacia el autofinanciamiento de recursos incorporadas en un nuevo modelo de negocios.

\section{Conclusiones.}

- Estas oportunidades de prosperidad, se ven perjudicadas por algunos aspectos como es el escaso reconocimiento y apoyo que encuentran estos espacios por parte del Estado, la poca accesibilidad a fuentes de financiamiento estables, limitada capacitación de los

- Los micro emprendimientos carecen de un registro financiero que detallen inversiones, ingresos y egresos, estructura financiera contable que logre un sistema coherente y coordinado entre el producto, el productor, el mercado y el ciclo del emprendimiento, que permita optimizar los recursos empresariales ampliando el crecimiento y la disminución de costos.

- Los bienes e infraestructura utilizada por los emprendedores en algunos casos son espacios alquilados que minimizan el tratamiento oficial de la logística motivacional elevando los costos de producción y el aprovechamiento tecnológico en el negocio, lo que permite la fidelidad de los clientes.

- Los micro emprendimientos conjugan la movilidad de recursos y la generación de riqueza, en base a la calidad de productos ofrecidos en un mercado específico de gastronomía, ocupando un punto crucial en la alimentación de la población, la diversidad, la presentación, la marca, el precio, son elementos desconocidos para algunos emprendedores que no brindan las seguridades del producto al cliente.

- El aporte que se brinda de la banca pública y privada, a la población en algunos casos son muy elevados y otros son escasos, disminuyendo las iniciativas productivas, este tipo de acciones condicionan los emprendimientos en magnitud y proliferación de negocios asociativos.

\section{Referencias bibliográficas.}

Villar, A. (2008). Políticas sociales en el área Metropolitana de Buenos Aires: viejos problemas de articulación intergubernamental y nuevos desafíos de gestión. In XIII Congreso Internacional del CLAD sobre la Reforma del Estado y de la Administración Pública. Buenos Aires, Argentina (Vol. 4). 
García, C. R., Puente, M. I., \& Saucedo, A. C. (2019). Aprovechamiento racional de los recursos naturales y culturales para el turismo comunitario, cantón Montalvo-Los Ríos. Ciencia Digital, 3(3), 129-141.

Cimoli, M., \& CEPAL, N. (2005). Heterogeneidad estructural, asimetrías tecnológicas y crecimiento en América Latina.

Torrent Sellens, J., \& Díaz-Chao, Á. (2014). ICT uses, innovation and SMEs productivity: Modeling direct and indirect effects in small local firms (No. WP14-001). IN3 Working Paper Series.

Olivos, P. C., Carrasco, F. O., Flores, J. L. M., Moreno, Y. M., \& Nava, G. L. (2015). Modelo de gestión logística para pequeñas y medianas empresas en México. Contaduría y administración, 60(1), 181-203.

Drucker, P. (1985). La Innovación y el Empresario Innovador. México: Edhasa.

Terán, H. E. E., Vargas, E. Y. M., Valle, J. A. B., \& Saltos, M. E. A. (2019). Análisis estructural y dinámica de los emprendimientos en la provincia de los ríos-zona norte. Universidad y Sociedad, 11(2), 104-108.

Salvador Borja, L. L. (2018). Nivel educativo y el emprendimiento empresarial en el centro de abastos de Lima Norte, 2017.

Borja López, J. C., \& DT Navas, E. (2015). Influencia de la Comunicación Publicitaria en el nivel de Posicionamiento de la Empresa" MAZ AVICULTURA" de la ciudad de Ambato (Bachelor's thesis, Universidad Técnica de Ambato. Facultad de Ciencias Administrativas. Carrera de Marketing y Gestión de Negocios.).

Ávalos-Monge, A. L., \& Murillo-Méndez, V. M. (2013). La necesidad de capacitación en planes de negocio en las microempresas de la zona de occidente de Costa Rica. InterSedes, 14(29), 56-80.

Jaramillo Villanueva, J. L., Morales Jiménez, J., Escobedo Garrido, J. S., \& Ramos Castro, J. G. (2013). Factores que influyen para el emprendimiento de microempresas agropecuarias en el Valle de Puebla, México. Revista mexicana de ciencias agrícolas, 4(spe5), 925-937.

Bustamante, K., \& Cabrera, K. (2017). Microcrédito, microempresa y educación en Ecuador. Caso de estudio: Cantón Zamora. Revista Espacios, 38(53).

Hegglin, D. R., Blanc, R. L., Ruhl, L. M., \& Cettour, W. H. (2013). Microcréditos y evolución de emprendimientos: experiencia en Entre Ríos.

Cimoli, M., Ferraz, J. C., \& Primi, A. (2005). Science and technology policies in open economies: The case of Latin America and the Caribbean.

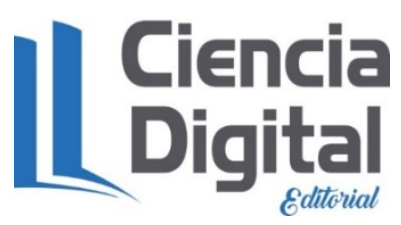




\section{Para citar el artículo indexado}

Rivera García, C., \& Parra Cerezo, G. (2020). La productividad y tipos de turismo en la provincia de Los Ríos. AlfaPublicaciones, 2(3), 43-54. https://doi.org/10.33262/ap.v2i3.34

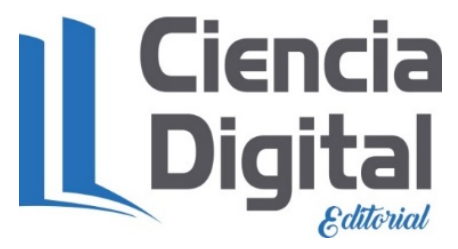

El artículo que se publica es de exclusiva responsabilidad de los autores y no necesariamente reflejan el pensamiento de la Revista Alpha Publicaciones.

El artículo queda en propiedad de la revista y, por tanto, su publicación parcial y/o total en otro medio tiene que ser autorizado por el director de la Revista Alpha Publicaciones.
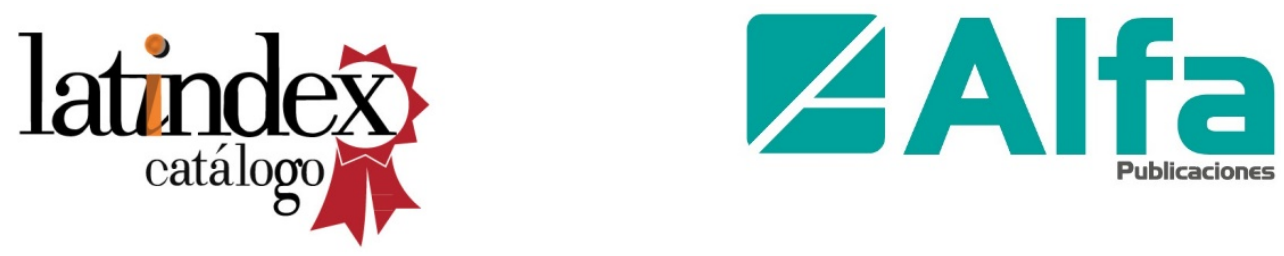